\title{
Relación bibliográfica de las traducciones de la obra de auguste comte a las principales lenguas del mundo.
}

\author{
Juan R. Goberna Falque \\ Universidad de Murcia \\ juangoberna@um.es (ESPAÑA)
}

La obra de Auguste Comte ha sido traducida desde el francés a al menos veinticuatro lenguas diferentes, un dato que por sí solo da cuenta de la importancia que esta actividad editorial ha tenido en la difusión del pensamiento comtiano a lo largo de la historia intelectual contemporánea. En este trabajo recopilatorio se pretende elaborar una relación lo más exhaustiva posible del conjunto de las versiones realizadas a partir de los escritos originales elaborados por el fundador del positivismo, así como de las principales antologías, resúmenes, etc. que nacieron al hilo de sus propios textos. Las principales lenguas de llegada han sido, como era de esperar, aquellas con un mayor peso en la historia intelectual del siglo XIX, XX y XXI, tales como el inglés, el alemán, el español, el portugués, el italiano, el ruso y el japonés, si bien la lista incluye también otras lenguas minoritarias, como el holandés, el catalán, el sueco, el danés, el polaco, el checo, el búlgaro, el húngaro, el rumano, el serbio, el croata, el macedonio, el griego, el turco, el chino o el coreano.

El protocolo de búsqueda de las referencias ha sido complejo, especialmente como consecuencia de los déficits que presentan todavía hoy en día los principales catálogos bibliográficos del mundo. Por citar sólo un ejemplo, en el Index Translationum, base de datos de la UNESCO dedicada a la historia de la traducción, únicamente aparecen 44 registros relativos a Comte como autor. Hemos realizado un vaciado sustancioso del catálogo de la European Library, que agrupa los de las 48 Bibliotecas Nacionales de Europa. Nos ha servido de gran ayuda también la información proporcionada por WorldCat, "The World Largest Library Catalog", en donde hemos podido seguirle la pista a las 1283 referencias bibliográficas. Con todo, el trabajo de cotejo, revisión y corrección ha sido muy intenso, como consecuencia de la profusión de errores catalográficos, y en muchas ocasiones las pesquisas han sido realmente arduas.

Una vez completada la relación completa de obras traducidas hemos procedido a su agrupación por lenguas de llegada, y dentro de cada una de ellas hemos realizado una ordenación en función del siguiente patrón: en primer lugar, aparecen sistemáticamente las antologías y obras misceláneas, esto es, aquellas en las que aparecen textos o fragmentos de textos procedentes de diversas obras de nuestro autor; a continuación, hemos hecho alusión a las traducciones realizadas para cada una de las obras por orden estrictamente cronológico de creación del texto en lengua origen (TLO), es decir, teniendo en cuenta la fecha 
de aparición del texto original en lengua francesa, y no la fecha de publicación de la traducción, que a efectos prácticos de la presentación de la información hemos considerado un dato de naturaleza secundaria. De modo que, por norma general, para cada una de las lenguas implicadas este ha sido el orden de exposición de las versiones realizadas : a) Opuscules primitifs (1819-1828); b) Cours de philosophie positive (1830-1842); c) Traité élémentaire de géométrie analytique à deux et à trois dimensions (1843); d) Discours sur l'esprit positif (1844) ; e) Discours sur l'ensemble du positivisme (1848, 1851); f) Calendrier positiviste ou Système générale de commemoration publique (1849); g) Système de politique positive, ou Traité de sociologie instituant la religion de l'Humanité (1851-1854); h) Catéchisme positiviste, ou Sommaire exposition de la religion universelle en treize entretiens systématiques entre une femme et un prêtre de l'humanité (1852) ; i) Bibliothèque positiviste (1854); j) Appel aux conservateurs (1855) y k) Synthèse subjective ou Système universel des concepcions propres à l'état normal de l'humanité (1856). Incluimos al final de cada serie la traducción de la correspondencia comtiana, así como la publicación de folletos y breves selecciones textuales.

\section{TRADUCCIONES EN LENGUA INGLESA}

Se puede asegurar, sin temor a error, que los lectores angloparlantes han estado siempre muy bien servidos de textos traducidos de la obra de Auguste Comte. Nombres como los de Harriet Martineau, Henry Dix Hutton, Frederic Harrison, Edward Spencer Beesly, John Henry Bridges o Richard Congreve son fundamentales para entender la difusión del positivismo comtiano, y todos ellos participaran intensamente en las labores editoriales y traductológicas relacionadas con la publicación de las versiones en lengua inglesa de las principales obras de nuestro filósofo. También es cierto que la mayoría de estas publicaciones son ciertamente antiguas, y quizás convendría revisar, actualizar o renovar por completo muchas de las traducciones. De hecho, algunos traductólogos sostienen que en el caso de los textos clásicos de la historia de la literatura o del pensamiento lo ideal sería realizar una nueva traducción de cada obra por cada generación, es decir, cada veinticinco años. En el caso que nos ocupa, el de las nuevas versiones en lengua inglesa de las principales obras del fundador del positivismo, apenas han destacado los proyectos editoriales ejecutados por Margaret Clarke, Gertrud Lenzer y H. S. Jones, por citar a los tres responsables de las publicaciones más recientes.

En efecto, la difusión del positivismo en el mundo anglosajón debe mucho a la extensa selección textual a partir del conjunto de la obra comtiana publicada por primera vez en 1975 bajo el título Auguste Comte and positivism: The essential writings (Nueva York, etc.: Harper Torchbooks. LXVIII, 505 p.). Esta obra, editada por Gertrud Lenzer, quien firma además el estudio introductorio correspondiente, presenta una amplia selección de extractos en cuatro grandes bloques temáticos: "Early Writings 1819-1828", "The First System: Cours de Philosophie Positive 1830-1842", "The Second System: Systeme de Politique 
Positive 1851-1854", "The Worship: Systeme de Politique Positive 18511854". La primera reimpresión data de 1983 (Chicago: University of Chicago Press) y en 1998 se publica otra edición de la misma obra (New Brunswick, NJ: Transaction Publishers. LXXXII, 642 p.), que incluye una nueva introducción y un postscript de la editora, este último tomado de la duodécima "Auguste Comte Memorial Trust Lecture", así como la «Conclusion of the whole work of the System of positive polity», procedente en este caso del volumen IV del Système de politique positive.

La primera versión en lengua inglesa de los opuscules primitifs sobre filosofía social data de 1877, fecha en la que Henry Dix Hutton elabora y publica el denominado "Appendix" que Comte había incluido en el último volumen del Système de politique positive. En efecto, esta traducción aparece por primera vez formando parte del volumen IV del System of positive polity or treatise on sociology: instituting the religion of humanity (Longmans, Green \& Co., 678 p.), y es publicada con el título "Theory of the future of man: with an appendix consisting of early essays on social philosophy". La relación de ensayos traducidos es la siguiente: "Separation of opinions from aspirations"; "A brief appraisal of modern history"; "Plan of the scientific operations necessary for reorganizing society"; "Philosophical considerations on the sciences and savants"; "Considerations on the spiritual power", e incluye un apéndice no incluido en la versión original en lengua francesa, titulado "Comte and Marx". La primera edición, ya en volumen separado, de esta primera traducción lleva por título Early essays on social philosophy (Londres, Nueva York, Dutton: George Routledge \& Sons. 352 p.), ve la luz en 1911, incluye notas adicionales y está encabezada por una introducción del editor, Frederic Harrison. En 1974, bajo el título ampliado The crisis of industrial civilization: the early essays of Auguste Comte (Londres: Heinemann Educational, [VI], 251 p.) se publica una reedición a partir de la traducción de Hutton de 1877, en esta ocasión a cargo de Ronald Fletcher, quien aporta una breve introducción. Y ya en 1998 se publica Early political writings (Cambridge; Melbourne: Cambridge university press. XLI-244 p.), dentro de la colección "Cambridge texts in the history of political thought", con edición y nueva traducción a cargo de H. S. Jones.

La más célebre traducción inglesa de cualquiera de las obras mayores de Comte es la que realiza en 1853 Harriet Martineau bajo el título The positive philosophy of Auguste Comte (Londres: John Chapman, 2 vols.: vol. 1: XXXVI, 480 p.; vol. 2: XVI, 561 p.; reed. facsimilar en 2001, Bristol: Thoemmes). En realidad, se trata de una versión muy libre, resumida y condensada del conjunto del Cours de philosophie positive, que incluye además una introducción realizada por Frederic Harrison. Las reediciones y reimpresiones de esta obra han sido muy frecuentes. Así, apenas un año después de la edición londinense, esto es, en 1854, aparece una versión idéntica en Estados Unidos (Nueva York: D. Appleton). Una nueva impresión de la obra tiene lugar en 1855 en la misma ciudad de Nueva York, pero ejecutada por una nueva editorial, Calvin Blanchard, (1 vol., XI, 838 p.; reimpr. en 1974 en Nueva York por AMS Press, encabezada por una nueva introducción a cargo de Abraham S. Blumberg). 
Hemos encontrado también una tirada aparte de una sección de esta obra, la correspondiente al resumen de los capítulos del Cours dedicados a la sociología, publicada bajo el título Social Physics: from the Positive Philosophy of Auguste Comte (Nueva York: Calvin Blanchard, 1856). En 1875 se reedita nuevamente en Londres (Trübner \& Co., $2^{\mathrm{a}}$ edición, otra vez en 2 vols.). En 1880 aparece una versión más, otra vez en un único volumen (Chicago; Nueva York [etc.]: Belford, Clarke \& Co., 838 p.; disponible en versión electrónica desde 2006). La tercera edición inglesa se publica en 1893 (S.l.: Kegan Paul, 2 vols.). En 1896 la obra consta por primera vez de tres volúmenes (Londres: G. Bell and Sons, vol. 1, 338 p.; vol. 2, 278 p.; vol. 3, 352 p.), reimpresos por el mismo editor en 1913, y cuya versión electrónica (Kitchener, Ont.: Batoche) está disponible desde 2000. En total, hemos localizado hasta doce ediciones diferentes de esta obra (ya sea en papel, ya sea electrónicas), cifra que por sí sola que da cuenta a la perfección del interés y la importancia que este resumen ha tenido en la transmisión del pensamiento comtiano y de la filosofía positivista en los países de lengua inglesa.

También contamos con una serie de publicaciones parciales o extractadas, pero no resumidas, de algunas de las lecciones del Cours. Así, en 1851 William M. Gillespie traduce y publica, a partir de las lecciones sobre filosofía matemática del primer tomo del Cours de philosophie positive, un volumen titulado The philosophy of mathematics (Nueva York: Harper \& Bros, XVI, 260 p.; reeditada en formato electrónico en 2012 al amparo del Project Gutenberg). En 1905 se publica The fundamental principles of positive philosophy: being the first two chapters of the "Cours de philosophie positive" (Londres: Watts \& Co. 63 p.). De la traducción se encargan Paul Descours y H. Gordon Jones y el volumen incluye un prefacio biográfico realizado por Edward Spencer Beesly. En 1970 se publica una Introduction to positive philosophy (Nueva York: BobbsMerrill, XIV, 70 p.) editada por Frederick Ferré. El volumen incluye una nueva introducción, a cargo de este editor, así como la traducción revisada de las dos primeras lecciones del Cours de philosophie positive. (reimp., Indianapolis: Hackett, 1988). Por último, en 1974 aparece The essential Comte (Londres: Croom Helm; Nueva York: Barnes and Noble Books, 252 p.), que en realidad constituye una selección de extractos pertenecientes al Cours de philosophie positive. De la edición y del estudio introductorio se encarga Stanislav Andreski, mientras que la traducción y las notas las realiza Margaret Clarke.

La primera versión inglesa del Discours sur l'esprit positif no se publica hasta 1883, bajo el título Preliminary discourse on the positive spirit, prefixed to the "Traite" philosophique d'astronomie populaire", traducción a cargo de Wathen Mark Wilks Call (Londres: Positivist Library, 154 p.). En 1903 aparece una nueva edición y traducción, en esa ocasión bajo el título abreviado de A discourse on the positive spirit, con traducción y notas a cargo de Edward Spencer Beesly (Londres: William Reeves, XXXIV, 172 p. (hemos localizado asimismo una edición electrónica de esta versión).

En 1865 ve la luz A general view of positivism: or summary exposition of the system of thought and life adapted to the Great Western Republic formed 
of the five advanced nations, the French, Italian Spanish, British and German which, since the time of Charlemagne have always constituted a political whole (Londres: Trübner \& Co. XII, 426 p.). Se trata de la traducción del célebre Discours sur l'ensemble du positivisme, cuya primera edición había sido publicada por Auguste Comte en París en 1848. John Henry Bridges realiza su versión a partir de la $2^{\mathrm{a}}$ ed., editada en 1851 como introducción al primer volumen del Système de politique positive, ou Traité de sociologie instituant la religion de l'Humanité. La edición de 1865 es reimpresa en papel en 1972 (Dubuque: Brown Reprints) y está disponible en formato electrónico desde 2006. Por otra parte, este texto, lo mismo que el TLO, encabeza el primer volumen del System of positive polity or treatise on sociology: instituting the religion of humanity (Londres: Longmans, Green \& Co.), publicado en 1875. Por otra parte, hemos de señalar que la traducción de Bridges ha sido reeditada en varias ocasiones con ligeras modificaciones respecto la primera edición inglesa de 1865. Así, en la edición de A general view of positivism publicada en 1908 (Londres: George Routledge \& Sons. XX, 444 p., in $8^{\circ}$ ) se incluye una introducción de Frederic Harrison, así como una serie de notas adicionales a partir de la edición francesa publicada en París en 1907. Hemos localizado hasta tres reimpresiones de esta edición: 1910 (Londres: s.e.); 1953 (Stanford: Academic Reprints); 1957 (Nueva York: Robert Speller and Sons, en este caso como edición conmemorativa del centenario a cargo del International Auguste Comte Centenary Committee).

En 1892 se edita por primera vez una versión en lengua inglesa, muy ampliada, del Calendrier positiviste, con el título The new calendar of great men: biographies of the 558 worthies of all ages and nations in the positivist calendar of Auguste Comte (s.1.: Macmillan and Co. 664 p.) En realidad, esta edición, a cargo de Frederic Harrison, incluye prolijas biografías de cada uno de los personajes incluidos originalmente por Comte en su Calendrier, de ahí la extensión del volumen. En 1894, el propio Harrison publica una edición idéntica a la del TLO, titulada en este caso The positivist calendar of 558 worthies of all ages and nations (Londres: W. Reeves. 16 p.). H. Gordon hace lo propio años más tarde, en 1929, año que el que aparece una nueva versión con el título The Positivist calendar of Auguste Comte and other tables (Londres: London Positivist Society, 48 p.).

Entre 1875 y 1877 se publica la traducción íntegra de los cuatro volúmenes correspondientes a la edición original del Système de politique positive, ou Traité de sociologie instituant la religion de l'Humanité. La versión inglesa se titula System of positive polity or treatise on sociology: instituting the religion of humanity (Londres: Longmans, Green \& Co.). El primer volumen lleva por título "General view of positivism \& introductory principles" (618 p.) y es traducido por John Henry Bridges; el segundo recibe el nombre de "Social statics, or the abstract theory of human order" (386 p.), y es traducido por Frederic Harrison; el tercero, por su parte, se llama "Social dynamics, or the general theory of human progress" (536 p.), y su traducción corre a cargo de Edward Spencer Beesly y otros, aunque de la revisión y edición final se 
encarga el propio Beesly; por último, el cuarto volumen, "Theory of the future of man: with an appendix consisting of early essays on social philosophy" (678 p.), es traducido básicamente por Richard Congreve (1818-1899), si bien, como hemos señalado más arriba, Henry Dix Hutton se encarga de la traducción del "Appendix" (Early essays). Este volumen culmina con un "Index" realizado por F. Harrison. Por otra parte, en 1890 se reimprimen los dos primeros capítulos de este vol. IV. en un volumen titulado The theory and worship of humanity (Liverpool: Liverpool Printing and Stationary Co., 158 p.) Habrá que esperar hasta 1968 y 1973 para que se publiquen sendas reimpresiones íntegras de la edición de 1875 (Nueva York: B. Franklin). Actualmente contamos con una edición electrónica del texto en lengua meta original (TLM), disponible desde 2006. Además, una breve selección de extractos del Système se recogen a su vez en un libro publicado en 1979 y titulado Social statics \& social dynamics: the theory of order and the theory of progress (Albuquerque, N.M.: American Classical College Press, 53 p.).

En 1858 se publica por primera vez The catechism of positive religion, or, summary exposition of the universal religion in thirteen systematic conversations between a woman and a priest of humanity (Londres: John Chapman. VI, 428 p.; edición electrónica disponible desde 2006). Como traductor consta Richard Congreve, una de las figuras señeras de la difusión de la obra comtiana en el mundo anglosajón. La $2^{\mathrm{a}}$ edición de esta versión, revisada y corregida, se publica en 1883 (Clifton, N.J.: Trübner, VIII, 315 p.), y la $3^{\mathrm{a}}$ y última, nuevamente revisada y corregida, aparece en 1891 (Londres: Kegan Paul, Trench, Trübner \& Co. VIII, 304 p.; reimpr. en 1973, Clifton: A. M. Kelley).

En 1886 ve la luz la traducción inglesa de la "Bibliothèque positiviste au dix-neuvième siècle", publicada originalmente en francés como apéndice del volumen IV del Système de politique positive. La versión en lengua inglesa aparece bajo el título The positivist library of Auguste Comte (Londres: Reeves \& Turner, 41 p.). En este caso, la traducción y edición corren a cargo de Frederic Harrison. Existe una reimpresión de esta obra, que data de 1971 (Nueva York: B. Franklin).

En 1889 se publica Appeal to conservatives (Londres: Trübner \& Co. 196 p.), con traducción a cargo de T. C. Donkin y Richard Congreve.

En 1891 se publica Subjective synthesis, or Universal system of the conceptions adapted to the normal state of humanity, etc. (Londres: Kegan Paul, Trench, Trübner \& Co. XL, 70 p.). El volumen incluye, como título de partida, Religion of Humanity y en la portada aparece la siguente mención: "First volume, containing the System of Positive Logic, etc.". Como traductor figura nuevamente Richard Congreve.

La correspondencia comtiana también ha sido objeto de traducción a la lengua inglesa. No abundan las realizaciones editoriales de este tipo, pero al menos hemos localizado algunas. El volumen Philosophical letter on marriage, by Auguste Comte (s.l.: s.e..), con traducción a cargo de Maria Congreve, es publicada en 1890. Poco después, en 1909, aparece Passages from the Letters of Auguste Comte (Londres: Adam and Charles Black, X, 209 p.), seleccionadas 
y traducidas por John K. Ingram. Más extensa es la obra titulada Confessions and testament of Auguste Comte and his correspondence with Clotilde de Vaux (Liverpool: Henry Young \& Sons. IX, 547 p.), edición a cargo de Albert Crompton, publicada en 1910. El último proyecto editorial de este tipo cuaja en 1995, año en el que se publica por primera vez en lengua inglesa el volumen The correspondence of John Stuart Mill and Auguste Comte (New Brunswick [u.a.]: Transaction Publ. XXVI, 403 p.), con edición y traducción a cargo de Oscar A. Haac e introducción de Angèle Kremer-Marietti.

Las circulares anuales dirigidas por Comte a sus correligionarios anglosajones también han sido publicadas. Hemos localizado una de ellas, bajo el título Voluntary fund instituted by Auguste Comte on behalf of the Priesthood of Humanity, 1. Annual circular addressed to every subscriber (Londres: Kenny. s.a.), pero de mayor interés resulta la relación completa, The eight circulars of Auguste Comte (Londres: Trübner \& Co. 89 p.), publicada en 1882, con edición de Richard Congreve y traducción a cargo de Samuel Lobb y otros. También habría que citar en este apartado algunos libritos divulgativos, como la breve selección textual que edita y publica este último autor, Samuel Lobb, en 1871, con el título A brief view of positivism (Calcuta: Thacker, Spink \& Co. VIII, 152 p.), o folletos muy genéricos, como Maxims, made or selected by A. Comte (Londres: A. Bonner, 4 p.), publicado en 1890 y editado por C. G. Higginson, o Golden words from Auguste Comte (Londres: Watts \& Co. 16 p.), una breve selección de textos editada en 1927 por Frederick James Gould. O incluso las lecturas públicas promovidas por el Auguste Comte Memorial Trust, las Memorial lectures 1953-1962 (Londres: The Athlone Press, University of London, 4 foll. correspondientes a la $1^{\mathrm{a}}, 2^{\mathrm{a}}, 3^{\mathrm{a}}$ y $5^{\mathrm{a}}$ lecturas, agrupadas en un vol. editado por la London School of Economics and Political Science). Para concluir, cabe referirse también en este apartado a la obra Before the second wave: gender in the sociological tradition (Upper Saddle River, NJ: Pearson Prentice Hall, págs. 3-12), publicada en 2006, en la que Barbara Finlay incluye un capítulo titulado "Auguste Comte (1798-1857) on women in positivist society", con extractos de consideraciones a este respecto realizadas por nuestro filósofo.

\section{TRADUCCIONES EN LENGUA ALEMANA}

A nadie debería sorprender descubrir que la lengua alemana sea la segunda lengua de llegada más prolija en publicaciones de traducciones de los textos comtianos, un empeño en el que participan en el transcurso del último siglo y medio autores como E. Roschlau, Wilhelm Ostwald, Valentine Dorn, Friedrich Blaschke o Jürgen Brankel. Con todo, y pese a su evidente fortaleza y posición de privilegio como consecuencia de la rica y variada gama de textos comtianos traducidos a dicha lengua, quizás también se podría subrayar alguna pequeña debilidad en la producción editorial en alemán a este respecto, como la sorprendente inexistencia, todavía hoy, de algún volumen antológico, una versión íntegra de los opuscules primitifs o de otros textos menores, como la 
Synthèse subjective, o de la propia correspondencia, completamente inédita.

En efecto, de los primeros opúsculos comtianos, sólo el Plan des travaux scientifiques nécessaires pour réorganiser la société ha sido traducido al alemán. Es en 1914 cuando el futuro Premio Nobel de Química Wilhelm Ostwald edita, introduce y anota el texto, que aparece publicado con el título Entwurf der wissenschaftlichen Arbeiten welche für eine Reorganisation der Gesellschaft erforderlich sind: (1822) (Leipzig: Verl. Unesma G.M.B.H., XV, 213 p.). Ya en 1973 se vuelve a imprimir la traducción editada por Wilhelm Ostwald, en esta ocasión con el título ligeramente modificado, Plan der wissenschaftlichen Arbeiten, die für eine Reform der Gesellschaft notwendig sind (Múnich: Carl Hanser Verlag, 156 p.), acompañado de una nueva introducción firmada por Dieter Prokop.

La primera versión en alemán de la que tenemos constancia del Cours de philosophie positive es la que ejecuta Georg Heinrich Schneider bajo el título Einleitung in die positive Philosophie (Leipzig: Fues's Verl. (R. Reisland), IV, 80 p.), pero en realidad se limita a la traducción de las dos primeras lecciones. Muy poco tiempo después, en 1883 y 1884, ven la luz sucesivamente los dos volúmenes de los que consta la obra Die positive Philosophie (Heidelberg: G. Weiss / Leipzig: Dürr, vol. 1: XXXI, 472 p.; vol. 2: X, [II], 524 p.), publicada originalmente en francés por Jules Rig (a la sazón, pseudónimo de JulesÉmile Rigolage) a partir de extractos tomados de los seis vols. del Cours, y traducida al alemán por Julius Hermann von Kirchmann. Entre 1907 y 1911 se acomete la traducción íntegra de los tres últimos volúmenes, que son editados en Alemania bajo el título genérico de Soziologie (Jena: Gustav Fischer) también en tres volúmenes: "Der dogmatische Teil des Sozialphilosophie" (vol. 1, 1907: XIX, 534 p.), "Historischer Teil der Sozialphilosophie: theologische und metaphysische Periode" (vol. 2, 1907: 570 p.) y "Abschluss der Sozialphilosophie und allgemeine Folgerungen" (vol. 3, 1911, XXXIV, 776 p.). De la ciclópea tarea traductiva se encarga Valentine Dorn, mientras que el estudio introductorio lo firma Heinrich Waentig. En 1923 se publica la $2^{\text {a }}$ edición de esta obra, bajo idénticas características editoriales. La última gran versión en alemán del Cours, nuevamente extractada, es la que edita Friedrich Blaschke en el año 1933 con el título Die Soziologie: die positive Philosophie im Auszug (Leipzig: Alfred Kröner Verlag, XXV, 563 p.). Apenas dos años después de la primera edición de la obra se publica la primera reimpresión, y ya en 1974 aparece una segunda edición de esta misma versión (Stuttgart: Kröner, XXXVII, 563 p.), que incluye una nueva introducción realizada por Jürgen von Kempski.

En 1915 se publica Abhandlung über den Geist des Positivismus: Ordnung und Fortschritt (Leipzig: F. Meiner, XVII, 140 p.), la primera de las versiones al alemán del Discours sur l'esprit positif, con edición y traducción a cargo de Friedrich Sebrecht. La segunda, publicada en 1956, lleva la firma de Iring Fetscher, autor asimismo del estudio introductorio que abre el volumen, y aparece bajo el título Rede über den Geist des Positivismus (Hamburgo: F. Meiner, XLV, 261 p.), con la particularidad de que en este caso se trata de una edición bilingüe. La obra se reimprime en 1966 y en 1979 de forma idéntica 
la edición original, hasta que en 1994 la editorial decide publicar una versión monolingüe de la misma (Hamburgo: Meiner, LII, 139 p.).

Contamos con una única versión en lengua alemana del Discours sur l'ensemble du positivisme, publicada en 1894 por E. Roschlau con el título Der Positivismus in seinem Wesen und seiner Bedeutung (Leipzig: Reisland, XII, [IV], 384 p.).

Entre 2004 y 2012 ven la luz los cuatro volúmenes correspondientes a la primera traducción alemana del Système de politique positive, ou Traité de sociologie instituant la religion de l'Humanité, un trabajo intelectual de características monumentales que debemos a Jürgen Brankel. La publicación, convenientemente anotada, lleva por título genérico System der positiven Politik (Viena: Turia + Kant) y consta, a diferencia del original, de cinco tomos, y además no incluye el apéndice del vol. IV del TLO con los opúsculos primitivos: vol. 1.1, "Widmung und Vorläufigen Diskurs" (2004: 390 p.); vol. 1.2: "Grundlegende, zugleich wissenschaftliche und logische Einführung" (2007: 296 p.); vol. 2: "Soziale Statik oder Die abstrakte Abhandlung über die menschliche Ordnung" (2008: 412 p.); vol. 3: "Soziale Dynamik oder Die allgemeine Abhandlung über den menschlichen Fortschritt" (2012: 526 p.) y vol. 4, 2012 (vol. 4: "Synthetische Tableau der Zukunft der Menschheit", 461 p.).

De la traducción del Catéchisme positiviste se encarga también E. Roschlau, quien en 1891 publica Katechismus der positiven Religion (Leipzig: Otto Wigand, VI, 405 p.), a partir de la $2^{\text {a }}$ edición del texto original.

Por último, hemos localizado asimismo una versión alemana del Appel aux conservateurs, realizada en 1928 por Eduard Lippmann con el título Aufruf an die Konservativen (Neufeld/Leitha: Lippmann; Viena: Jahoda und Siegel, XXXIX, 133 p.).

\section{TRADUCCIONES EN LENGUA ESPAÑOLA}

En la producción editorial de traducciones de obras de Auguste Comte en español sobresale en un primer momento el papel de divulgación emprendido por editoriales hispanoamericanas (Chile, Argentina y México, sobre todo), si bien desde los años 80 del pasado siglo es España el país en el que se produce el mayor número de publicaciones. El conjunto de la obra comtiana está bien representado en lengua española, aunque se han dado casos en los que se ha producido una importante sobreproducción (como es el caso de las sucesivas versiones y reediciones del Discours sur l'esprit positif) que no logra ocultar significativas lagunas, como por ejemplo la ausencia de una traducción parcial o total del Sistème de politique positive o del Appel aux conservateurs. Por citar algunos de los nombres de referencia en esta tarea de divulgación del positivismo comtiano, quizás habría que hacer alusión en este momento al trabajo traductológico llevado a cabo por Jorge y Luis Lagarrigue, Antonio Zozaya, Maximio S. Victoria, Nicolás Estébanez, Francisco Giner de los Ríos, Julián Marías, Andres Bilbao o quien estas páginas subscribe, Juan R. Goberna Falque. 
La única antología que hemos localizado en lengua española se publica en Argentina en 1943, lleva por título Comte: selección de textos (Buenos Aires: Editorial Sudamericana, 223 p.; existe edición digital), con traducción y notas a cargo de Demetrio Náñez y un estudio introductorio de René Hubert.

Francisco Giner de los Ríos realiza una traducción al español de los Primeros ensayos de Auguste Comte, que publica en 1942 en México (México: Fondo de Cultura Económica, VIII, 303 p.; reimpr. en 1997 y 2001). El Plan des travaux scientifiques nécessaires pour réorganiser la société (1822) se edita en 2000 en castellano, con traducción de Cristina B. Negro Konrad, bajo el título Plan de los trabajos científicos necesarios para reorganizar la sociedad (Madrid: Tecnos, XL, 135 p.; existe edición digital de la obra) e incluye un estudio preliminar a cargo de Dalmacio Negro Pavón.

La traducción parcial o resumida al español del Cours de philosophie positive es relativamente significativa, sobre todo en comparación con lo que ha sucedido en otras lenguas. Así, hemos de señalar que en 1895 ven la luz en Argentina los dos volúmenes de los que consta la obra La filosofía positiva (Buenos Aires: Imprenta "El Hogar y la Escuela", 2 vols. in 1, 400 p.), la cual, como ya hemos visto, había sido publicada originalmente en francés por JulesÉmile Rigolage. Firma la traducción Maximio S. Victoria. El volumen 1 incluye resúmenes de la "Introducción" y la "Matemática", mientras que en el volumen 2 se extracta la "Física", la "Química", la "Biología" y la "Sociología" del Cours. Contamos además con al menos tres versiones al español de las dos primeras lecciones del Cours: la más antigua (Santiago de Chile: Imprenta de la Librería del Mercurio, 1875, 182 p.) es la de Jorge Lagarrigue, primer traductor al español de los Principes de philosophie positive (París: J. B. Baillière et fils, 1868), el volumen póstumo en el que se incluyen precisamente estas dos primeras lecciones, amén de un prefacio de Émile Littré. Hemos localizado dos ediciones de esta obra en Centroamérica (Guatemala: Tip. Americana, 1895; Nicaragua: Tip. Mercatil de Pedro Argüello, 1901), sin mención de la autoría de la traducción. Por su parte, Luis de Terán se encarga poco después de hacer otra versión de esta misma obra, hacia 1890, también bajo el título Principios de filosofía positiva (Madrid: La España Moderna, 142 p.) y que fue reeditada en los años treinta. Una versión algo más reciente de las dos primeras lecciones en la que aparece en un pequeño volumen titulado Curso de filosofía positiva. Lecciones I y II (Buenos Aires, Aguilar, 1973. 113 p.), prologado y anotado por José Manuel Revuelta, quien se encarga además de la traducción (y que cuenta con numerosas reediciones, entre otras, Buenos Aires: Aguilar, 1981, 113 p.; Barcelona: Orbis, 1984, 1985, 189 p.; Barcelona: Folio, 1999; 2002, 191 p.). También disponemos en castellano de una traducción íntegra de las lecciones $46^{\mathrm{a}}$ y $47^{\mathrm{a}}$, publicadas en un volumen titulado La física social (Madrid: Aguilar, 1981, LI, 273 p.), prologada, traducida y anotada por Dalmacio Negro Pavon. Por último, quisiera destacar, obviamente, la publicación en 2012 de las doce lecciones del CFP (46 ${ }^{\mathrm{a}}-57^{\mathrm{a}}$, ambas incluidas) correspondientes a la Física social (Madrid: Akal, 1295 p.), con estudio introductorio, traducción y notas de quien este trabajo suscribe, Juan R. Goberna Falque, que a mi modo 
de ver ha colmado un sustancial vacío editorial e intelectual en el ámbito de la difusión del pensamiento comtiano dentro de la comunidad lingüística y cultural hispanófona.

Existen al menos cinco traducciones diferentes del Discours sur l'esprit positif en castellano: la primera fue la famosa versión editada en la Revista de Occidente en 1934, con el título «Discurso sobre el espíritu positivo», traducida y prologada por Julián Marías, y que ha contado con numerosas reediciones (Madrid: Alianza Editorial, 1980, 1984, 1988, 1997, 2000, XL, 135 p.; Barcelona, Altaya, 1995; etc.). En 1936, apenas dos años después de la publicación en España primera versión en español del Discours, se edita en Argentina una nueva traducción (Buenos Aires: Imp. La Vanguardia, 203 p.), firmada en este caso por Maximio S. Victoria, introducida por "un preámbulo de interpretación por Emilio Corra expuesto por Alfredo J. Ferreira en la $4^{a}$ sesión del Comité positivista argentino el 19 de octubre de 1924", según consta en los títulos de crédito de la obra. Arístides Gregori publica en 1940 otra traducción, con el título El espíritu positivo (Buenos Aires: Tor, 130 p.). Consuelo Berges, por su parte, también realiza a principios de los años cincuenta una nueva traducción del texto (Buenos Aires, Madrid, [etc.]: Aguilar, 1953, 170 p.; numerosas reimpr.: 1961, 1962, 1965, 1971, 1980, 1982,; existe versión en formato digital), con prologo de Antonio Rodríguez Huéscar, y además reeditada en España en un volumen titulado Discurso sobre el espíritu positivo (Madrid: Sarpe, 1984, 157 p.) en el que también se incluye la traducción de José Manuel Revuelta, anteriormente citada, de las dos primeras lecciones del Curso de Filosofía Positiva; y, por último, Eugenio Moya ha publicado asimismo una quinta versión del mismo texto, con el título Discurso sobre el espíritu positivo: discurso preliminar del tratado filosófico de astronomía popular (Madrid, Biblioteca Nueva, 1999, 164 p.), encargándose además de la introducción y de las notas.

Del Calendrier positiviste hemos localizado una única traducción, fechada en 1884, con el título homónimo de Calendario positivista (Santiago de Chile: Imprenta Cervantes, 28 p.), ejecutada probablemente por Jorge Lagarrigue.

En cambio, existen cuatro versiones en castellano del Catéchisme positiviste: la primera, muy antigua, fue la que realizó el periodista y filósofo Antonio Zozaya bajo el título Catecismo positivista o sumaria exposición de la religión universal en trece diálogos sistemáticos entre una mujer y un sacerdote de la Humanidad (Madrid, Biblioteca Económica Filosófica, 1886-1887; vols. 30, 31 y 32, reeds. en 1899 y 1920); la segunda, firmada por el ideólogo federalista Nicolás Estébanez, y publicada en 1913 con el título truncado Catecismo positivista, o Exposicion sumaria de la religión universal (París: Garnier, 346 p.; reed. en 1920); la tercera, la que realiza Luis Lagarrigue, es publicada en Chile en 1938 con el título La religión universal: doce diálogos entre Clotilde de Vaux y Augusto Comte (Santiago de Chile: Soc. Imp. y Lit. Universo, 218 p.; edición digital, s.l., HathiTrust Digital Library, 2010) y por último, la cuarta versión, ya más reciente, es la que presenta y anota Andrés Bilbao (Madrid, Editora Nacional, 1982, 299 p.). 
Asimismo, en el trascurso de nuestros rastreos catalográficos hemos localizado una edición en castellano de la Synthèse subjective, publicada en 1944, a cargo de Luis Lagarrigue, y titulada Síntesis subjetiva o Sistema Universal de las concepciones propias al Estado Normal de la Humanidad: bosquejo del Sistema de Industria proyectado por el Supremo Maestro Augusto Comte (Santiago de Chile: Fundación Juan Enrique Lagarrigue, 385 p. —versión digital: s.l.: HathiTrust Digital Library, 2010_; reed. en 1946, 271 p.).

En cuanto a los folletos y otras selecciones de extractos comtianos, habría que señalar que en 1978 se edita en México La teoría social del positivismo (México: Centro de Documentación Política, 77 p.), una obrita constituida por una breve selección de textos traducidos por Georgette José Valenzuela y agrupados en una serie de capítulos tales como "Decadencia de los antiguos sistemas políticos", "La crisis social", "La nueva política", "Estática social o teoría del orden", "Teoría general del progreso o dinámica social" y "El régimen positivo. Organización y funcionamiento". También en México, en 1979, se publica Ensayo de un sistema de política positiva (México: Universidad Nacional Autónoma de México, 140 p.; disponible en versión digital), acompañado de un estudio preliminar firmado por Raúl Cardiel Reyes.

\section{TRADUCCIONES EN LENGUA PORTUGUESA}

La gran difusión del positivismo en Brasil en el transcurso del último cuarto del siglo XIX y los primeros años del siglo XX entre las élites políticas e intelectuales de este país sudamericano explican la gran profusión de traducciones de las diferentes obras de Auguste Comte, especialmente significativa en el caso de la edición de folletos y pequeñas selecciones temáticas, así como en la traducción de parte de la correspondencia comtiana, realizadas por obra y gracia de Miguel Lemos y Raymundo Teixeira Mendes o Dinarte Ribeiro. Lejos de caer en el olvido, en el último cuarto del siglo XX florecen nuevamente en Brasil, e incluso en Portugal, los continuadores de dicha tradición, como lo pone de manifiesto la rica labor traductológica llevada a cabo por José Arthur Gianotto, Ivan Lins o Evaristo de Moraes Filho. Con todo, se sigue echando muy en falta una traducción más completa en lengua portuguesa de las dos obras mayores de Auguste Comte, tanto de Cours de philosophie positive (limitada, de momento, a las dos lecciones introductorias), así como del Système de politique positive, totalmente inédita.

Desde luego, en lengua portuguesa abundan las antologías de textos comtianos. En 1978 se publica Comte: os Pensadores (São Paulo: Abril Cultural, XVI, 318 p.) una obra miscelánea que incluye la traducción de varias de sus obras de referencia. El volumen, encabezado por un estudio biográfico del fundador del positivismo, acompañado de una cronología y una bibliografía, contiene las dos primeras lecciones del Curso de filosofía positiva ("Primeira Lição: Exposição da finalidade deste curso, ou considerações gerais sobre a natureza e a importância da filosofia positiva"; "Segunda Lição: Exposição do plano deste curso, ou considerações gerais sobre a hierarquia das ciências 
positivas"), las tres partes del Discurso sobre o espírito positivo ("Primeira Parte: O espírito positivo é mais apto para organizar a harmonia mental do que o espírito teológico-metafísico"; "Segunda Parte: O espírito positivo é mais apto que o espírito teológico-metafísico para organizar a sociedade e sistematizar a moral"; "Terceira Parte: Condições do estabelecimento do regime positivo", el preámbulo general y la primera parte del Discurso preliminar sobre o conjunto do positivismo ("Espírito fundamental do positivismo"), todos ellos traducidos por José Arthur Giannotti, así como el Catecismo positivista íntegro, traducido y anotado por Miguel Lemos. La $2^{\mathrm{a}}$ edición (São Paulo: Abril, 1983) es idéntica a la primera, y las siguientes presentan leves modificaciones (São Paulo: Nova Cultural, 1988, reimpr. en 1991, XVI, 264 p.; São Paulo: Nova Cultural, 1996, reimpr. en 2000, 336 p., no incluyen el Discurso sobre o espírito positivo). Por otra parte, también en 1978 se edita por primera vez una compilación de textos sociológicos comtianos en una obra titulada Auguste Comte: sociologia (São Paulo: Ática, 208 p.; reed. en 1983 y 1989), seleccionados y traducidos por Evaristo de Moraes Filho, quien firma además la introducción de la obra. Por último, cabe citar la publicación en Portugal, en 1984, de un volumen titulado $O$ espirito positivo (Oporto: RES, XVII, 237 p.) y que incluye versiones del Plan des travaux scientifique pour reorganiser la société, el Discours sur l'esprit positive y el Appel aux conservateurs, con introducción, selección textual y notas a cargo de Maria Luiza Borralho y traducción de Carlos Lopes Monteiro.

Pasando ya a la relación cronológica de traducciones completas o parciales de las diferentes obras de Auguste Comte, hemos de indicar en primer lugar que desde 1899 existe una versión íntegra de sus primeros escritos merced a una compilación titulada Opúsculos de Filosofia Social: 1819-1828 (Porto Alegre: Livraria do Globo, LIV, 352 p.), con traducción a cargo de Dinarte Ribeiro. Ya en 1972 se publica una nueva adaptación de esta obra (Porto Alegre: Globo; São Paulo: USP, XX, 233 p.), con traducción a cargo de Ivan Lins y João Francisco de Souza, e introducción de Sofia Beatriz Lins Peixoto. Como acabamos de ver, una versión del Plan des travaux, ejecutada por Carlos Lopes Monteiro, ha sido incluida en el volumen antológico O espírito positivo (Oporto: RES, XVII, 237 p.). Este mismo opúsculo ha sido traducido también al portugués con el título Reorganizar a sociedade (Lisboa: Guimaräes, 196 p.; reed. en 1990, 1993 y 2002), con prefacio y traducción a cargo de Álvaro Ribeiro.

Hemos localizado únicamente tres referencias bibliográficas directamente relacionadas con la traducción al portugués de las dos lecciones introductorias del Cours de philosophie positive. La primera de ellas es Principios de filosofía positiva (São Paulo: Editorial Paulista, 1876, 178 p.), una versión más, en este caso brasileña, de los Principes de philosophie positive, editados póstumamente, como ya hemos visto en el epígrafe precedente, en París en 1868, con la reproducción íntegra de las dos primeras lecciones del Cours; la segunda data de 1883, responde al mismo plan editorial que la anterior, y se publica en dos volúmenes con el título prácticamente homónimo de Principios de philosophia positiva, extrahidos do Curso de philosophia positiva (Oporto: s.e.; existe edición digital de la obra), con edición a cargo de Teixeira Bastos; y la tercera 
es la que se edita en 1973 en la obra Curso de filosofia positivista (São Paulo: Abril Cultural; reimpr., formando parte del volumen antológico Comte: os Pensadores, 1978, ya citado), con traducción de José Arthur Giannotti, si bien una vez más se limita a la versión portuguesa de la $1^{\mathrm{a}}$ y la $2^{\mathrm{a}}$ lecciones del Cours.

La versión portuguesa del Traité élémentaire de géométrie analytique à deux et à trois dimensions puede considerarse excepcional, pues constituye la única traducción que ha conocido esta obra en cualquier lengua. Miguel Lemos y Raymundo Teixeira Mendes realizan en 1875 una traducción del texto, Geometria analytica (Río de Janeiro: Typ. Academica, 31 p.), que no llega a circular porque finalmente consideran más oportuno reeditar el texto en francés. Apenas seis años después, en 1881, ve la luz una segunda versión, publicada por el Club Academico da Escola Militar de Río de Janeiro, bajo el título Tratado elementar de geometria analytica a duas e três dimensoes (Río de Janeiro: Club Academico da Escola Militar, 32 p.).

En 1947 se edita una versión portuguesa del primer Discours, la cual lleva por título Discurso sobre o espírito positivo (Lisboa: Seara Nova, 152 p.), con traducción, introducción, tabla cronológica y sincrónica y notas a cargo de Joel Serrão. En Brasil se edita, ya en 1973, una nueva versión de esta misma obra (São Paulo: Abril Cultural), traducida en esta ocasión por José Arthur Giannotti. Apenas tres años después, en 1973, ve la luz una nueva edición (Porto Alegre: Globo; São Paulo: Editora da Universidade de São Paulo, 130 p.), con traducción a cargo de Renato B. R. Pereira y revisión de Ivan Lins. En 1990 se publica una cuarta versión en portugués de la obra, con traducción a cargo de Maria Ermantina Galvão Gomes Pereira y revisión de Roberto Leal Ferreira (São Paulo: Livraria Martins Fontes, 132 p.). Y por último, ya en 2005 , se publica la quinta y de momento más reciente traducción al portugués del Discours (São Paulo: Escala, 110 p.), ejecutada en este caso por Antonio Geraldo da Silva.

En cuanto se refiere a las versiones en lengua portuguesa del segundo Discours, ya hemos señalado que tanto el preámbulo como la primera parte del Discurso preliminar sobre o conjunto do positivismo ("Espírito fundamental do positivismo"), forman parte del volumen antológico titulado Comte: os pensadores (cf. supra), publicado por primera vez en 1978 y traducido por José Arthur Giannotti (São Paulo: Abril Cultural, XVI, 318 p.). Además, un extracto de la quinta parte de este mismo Dircours se publica en 1949 en un folleto aparte bajo el título Aptidão estética do positivismo (Río de Janeiro: Ed. A Noite, 42 p.), con traducción a cargo de F. A. Machado da Silva.

La primera vez que se publica una versión portuguesa del Calendrier positiviste es en 1877, fecha en la que Miguel Lemos publica una obrita titulada Pequenos ensaios positivistas (Río de Janeiro: Brown \& Evaristo, 161 p.), en la que además se recopilan diversos artículos de este autor publicados originalmente en diversos periódicos brasileños. La misma tabla se vuelve a publicar en 1885, formando parte en esta ocasión de una antología de textos comtianos que lleva por título $O$ Calendario positivista: seguido da Bibliotheca positivista: e precedido de indicações geraes sobre o positivismo (Río de 
Janeiro: Publicações Centro Positivista, 95 p.), en la que Miguel Lemos también incluye una versión en lengua portuguesa de la Bibliothèque positiviste, así como una traducción del texto "Indicações geraes sobre o positivismo", de Henry Edger, publicado originalmente en lengua inglesa. En 1899 Raymundo Teixeira Mendes edita la obra Calendario positivista: precedido de indicaçoes summarias sobre a theoria pozitiva do calendario (São Paulo, F. M. Germano, 51 p.), En 1902 se reedita una versión de la compilación de Miguel Lemos de 1885, con el título ligeramente modificado: Calendário e bibliotéca pozitivistas: seguidos respetivamente de um índice onomástico e de um índice bibliográfico (Río de Janeiro: Séde Central da Igreja Pozitivista do Brazil, VIII, 71 p.), pero que en lo sustancial reproduce el contenido del volumen publicado en 1885 .

En 1890 Miguel Lemos publica su primera traducción del Catéchisme, que ve la luz en un volumen titulado Catessismo pozitivista: o sumária espozisao de relijiao universal en 13 conferensias sistemáticas entre uma mulhér $i$ un sasserdóte da Umanidade (Río de Janeiro: s.e., 351 p.). La versión, anotada por el propio Miguel Lemos, tendrá en el curso de los siguientes años tres ediciones más, publicadas respectivamente en 1895 (Río de Janeiro: Capela da Humanidade, VII, 536 p.), 1905 (Río de Janeiro: Igreja pozitivista do Brazil, 497-II-39 p.) y 1934 (Lisboa; Río de Janeiro: Lucas \& Cia; Igreja Pozitivista do Brazil, $4^{\mathrm{a}}$ ed., conforme a la $3^{\mathrm{a}}$ ). Incluye un "Indice alfabetico das materias contidas no Catecismo Pozitivista de Augusto Comte" elaborado por Jozé Mariano de Oliveira. Ya en 1973 se publica una nueva reedición de esta misma publicación (São Paulo: Abril Cultural). Y algo más reciente es la edición de una nueva versión de esta misma obra bajo el título Catecismo positivista ou exposição sumária da religião universal de onze colóquios sistemáticos entre uma mulher e um sacerdote da humanidade (Lisboa: Lisboa, Publicações Europa-América, [1975], 258 p.), con traducción a cargo de Fernando Melro.

También contamos con una traducción portuguesa del Appel aux conservateurs, a cargo nuevamente de Miguel Lemos, gran divulgador brasileño de la obra y el pensamiento comtiano, que aparece en 1899 con el título Apelo aos conservadores (Río de Janeiro: Igreja Pozitivista do Brazil, XXXV, 250 p.).

Por último, debemos hacer mención a la publicación de una traducción parcial de la Synthèse subjective ou Système universel des concepcions propres à l'état normal de l'humanité, titulada en este caso Introduçao Geral ao Estudo da Lógica, ou Matemática, traducida y anotada por Luiz Bueno Horta Barbosa (Río de Janeiro: Tipografia do Jornal do Commercio, 1933).

En el conjunto de la bibliografía traducida de la obra comtiana, sorprende de algún modo la relativamente significativa cantidad de obras publicadas en portugués en las que se recoge parcialmente su correspondencia. Hemos localizado cinco publicaciones de este tipo, todas ellas pertenecientes al periodo álgido del positivismo brasileño: Miguel Lemos se encarga de la traducción de la Carta filozófica sobre a comemoração social (Río de Janeiro: Igreja Pozitivista do Brazil, 21 p.), publicada en 1896 dentro de la serie "Apostolado pozitivista do Brazil", no 168. Entre 1897 y 1898 ve la luz Romance de Augusto Comte: excerptos das obras e correspondencia do incomparavel mestre 
(Pernambuco: Typographia do "Jornal do Recife"), una obra compilada y traducida por A. Pereira Simões en dos volúmenes (vol. 1: "Augusto Comte"; vol. 2.: "Clotilde de Vaux", versión digital en [S.1.]: HathiTrust Digital Library, 2010). Bajo la dirección editorial de Raymundo Teixeira Mendes se publica en 1900 el volumen $O$ ano sem par: abril de 1845 a abril de 1846, ou, Meditação religiosa da incomparavel união á qual os fundadores do positivismo, Augusto Comte e Clotilde de Vaux (n. Marie), deverão o preenchimento da sua missão: esboço (Río de Janeiro: Templo da Humanidade, XIII, 984, 64 p.; edición digital: [S.1.]: HathiTrust Digital Library, 2010). En 1903 (i.e. 1936) se edita en portugués la obra Comte e Clotilde (Río de Janeiro: Ed. Filial, 126 p.) en la que se recogen textos como "Lúcia", "Os pensamentos de uma flor", "A infáncia", "Carta sobre a comemoração social" y "Orações quotidianas". Por último, en 1912 se edita la obra Cartas filozóficas sobre a comemoração social, o batismo cristão, o cazamento (Porto Alegre: Propaganda Positivista, 53 p.) y también el Manifesto inicial da sociedade positivista de Paris. O fundador da sociedade positivista a todos aqueles que desejem se incorporar nela (Porto Alegre: s.e., 15 p.), ambos traducidos una vez más por Miguel Lemos y Raymundo Teixeira Mendes. Por otro lado, F. A. Machado da Silva traduce y edita en 1945 Algumas cartas inéditas de Auguste Comte (Rio de Janeiro: Jornal do Comércio, 25 p.) y desde 2002 contamos con la publicación de la correspondencia entre Comte y la positivista brasileña Nísia Floresta Brasileira Augusta, en una obra editada por Constância Lima Duarte y que lleva por título Nísia Floresta \& Auguste Comte (Florianópolis; Santa Cruz do Sul, RS: Editora Mulheres; EDUNISC, 2002, 101 p.; existe edición digital de la compilación), elaborada a partir de las traducciones realizadas por Miguel Lemos y Paula Berinson.

Tenemos que hacer alusión asimismo a la gran cantidad de folletos y breves selecciones de textos comtianos publicados en portugués, especialmente como consecuencia de las actividades de difusión llevadas a cabo por los positivistas brasileños. La primera traducción al portugués de la que tenemos constancia de algún texto relacionado con la obra de Auguste Comte es un folio plegado titulado "Classificassão pozitiva das dezoito funsões interiores do sérebro, ô cuadro sistemático da alma", editado en París por el Apostolate positiviste entre 1848 y 1849. En 1880 Joaquim Ribeiro de Mendonça, a la sazón presidente da Sociedade Positivista do Rio de Janeiro, publica Circulares do Fundador do Positivismo, traducción de las circulares de Augusto Comte, ilustrada con un retrato del filósofo (São Paulo: Tipografia da Província, 120 p). De este mismo autor es la obrita Do espirito positivo, (São Paulo: Abílio S. Marques, 83 p.), publicada en 1880 a partir, indica, de las "notas recopiladas por un discípulo en los cursos de Augusto Comte". En 1884 Miguel Lemos publica su traducción de una selección de textos comtianos que él mismo realiza relativos a la cuestión esclavista: $O$ pozitivismo e a escravidão modérna: trechos extraídos das óbras de Augusto Comte, seguidos de documentos pozitivistas relativos à questão da escravatura no Brazil e precedidos de uma introdução (Río de Janeiro: Soziedade Pozitivista, 66 p.; texto microfilmado en Nueva York: New York Public Library, 1977). En 1895 Raymundo Teixeira Mendes 
publica Programas de ensino (Río de Janeiro: [s.n.]), un texto de 36 páginas elaborado a partir de los puntos de vista comtianos relacionados con el ámbito de la educación. En 1896 se publica una traducción de Miguel Lemos de la "Lettre philosophique sur la commemoration sociale", con el título Carta filozofica sobre a comemoracão social (Río de Janeiro: Igreja Pozitivista do Brazil, 21 p.). Lemos traduce y publica asimismo en 1900 el Manifesto inicial da Sociedade pozitivista de Paris (Río de Janeiro: Igreja Pozitivista do Brazil, 50 p.). En 1910, Raymundo Teixeira Mendes publica en el Jornal do comércio una serie de artículos que posteriormente edita en un pequeño folleto de diez páginas titulado Atitude dos pozitivistas ante a retrogradação militarista (Rio de Janeiro: Apostolado pozitivista do Brazil), con textos extraídos de vol. IV de la Politica pozitiva de Augusto Comte. En ese mismo año 1910 se edita Clotilde: les pensées d'une fleur, l'enfance, les sept maximes (Rio de Janeiro: Igreja Pozitivista do Brazil, 12 p.), una edición bilingüe francés-portugués de dos poemas de Auguste Comte a Clotilde de Vaux también realizada por Raymundo Teixeira Mendes, quien además anota el texto e incluye una oración por el filósofo positivista. En 1915 se publica el esbozo biográfico que Auguste Comte había hecho en su día de Teresa de Avila en un folleto titulado Santa Tereza: 28 de março de 1515 - 15 de outubro de 1582: sua glorificação social (Río de Janeiro: Igreja Pozitivista do Brazil, 15 p.). Ya en 1936 ve la luz un breve folleto titulado Mássimas pozitivistas (Río de Janeiro: Pelos, 20 p.). En 1939 se publica el librito Importância da filosofia positiva (Lisboa: Editorial Inquérito, 72 p.; $2^{a}$ ed., 1950, 69 p.; reimpr. en 1961), con traducción a cargo de J. A. Freitas e Silva.

\section{TRADUCCIONES EN LENGUA ITALIANA}

La característica más sobresaliente de la edición de traducciones comtianas en lengua italiana tiene que ver con el tardío despegue del proceso. En efecto, la primera versión de un texto de Auguste Comte en italiano se publica en 1882, pero prácticamente habrá que esperar hasta los años sesenta del pasado siglo para que empiecen a proliferar realmente en Italia las traducciones del resto de sus obras. La labor traductológica emprendida desde entonces permite a cualquier lector italiano el acceso a un buen número de publicaciones originales del fundador del positivismo en su lengua materna, lo cual, sin duda, ha permitido una mejor comprensión del conjunto de la filosofía comtiana. Desde luego, si hubiera que destacar algunos nombres en particular por su papel en la divulgación del pensamiento de Auguste Comte en Italia tendríamos que hacer alusión sin duda alguna a Antimo Negri, en primerísimo lugar, así como a Franco Ferrarotti, Emanuele Riverso y Almerino Lunardon. Muy completas, y variadas, son las versiones italianas de los primeros opúsculos, del propio Cours y también de los dos Discours, pero, una vez más se percibe una laguna significativa en lo que se refiere a la traducción del Système de politique positive, o incluso en las de algunas de las obras menores, tales como el Appel aux conservateurs o la Sinthèse subjective, que todavía no han sido acometidas. 
En 1966, Antimo Negri edita una relativamente extensa antología de textos extraídos del Cours de philosophie positive, el Discours sur l'esprit positif y el Systeme de politique positive en una obra que lleva por título Filosofia positiva (Florencia: G. C. Sansoni, LXXXIII, 332 p.), ocupándose además de la introducción, la traducción y las notas. Apenas tres años después, en 1969, este mismo autor se encarga de la edición, introducción y traducción de los Opuscoli di filosofia sociale e discorsi sul positivismo (Florencia: G. C. Sansoni, XLVIII, 787 p.), que contiene las versiones íntegras al italiano de los Opuscules primitifs sur la philosophie sociale, el Discours sur l'esprit positif y el Discours preliminaire sur l'ensemble du positivisme. Asimismo, ya en 1977, se publica Antologia di scritti sociologici (Bolonia: Il Mulino, 197 p.), a cargo de Franco Ferrarotti, el cual, como reza el propio título, constituye una selección de extractos tomados de diferentes textos sociológicos comtianos.

La primera traducción del Plan des travaux scientifiques nécessaires pour réorganiser la société es la que Enrico Vidal incluye en 1959 como apéndice de su obra Saint-Simon e la scienza política (Milán: A. Giuffrè, pp. 145-234) con el título "Sistema di politica positiva di Auguste Comte". Además, tal y como acabamos de ver, existe una versión íntegra en lengua italiana de los primeros opúsculos inserta en la obra editada en 1969 por Antimo Negri Opuscoli di filosofia sociale e discorsi sul positivismo (Florencia: G. C. Sansoni, XLVIII, 787 p.).

Entre 1964 y 1974 se publican en Italia hasta cinco versiones diferentes, más o menos amplias, del Cours de philosophie positive. En 1964 se traducen las dos primeras lecciones, editadas en un pequeño volumen titulado Filosofia e scienze: preliminari generali della filosofia positiva: 1. e 2. lezione del Corso di filosofia positiva di Augusto Comte (Turín; Roma; Nápoles: Ist. editoriale del Mezzogiorno, 111 p.; existe versión digitalizada de la obra), con traducción, introducción y notas a cargo de Emanuele Riverso. Apenas dos años después, en 1966, se editan algunos extractos del Cours en el volumen antológico Filosofia positiva publicado por Antimo Negri, ya citado. En 1967 se publica otra breve selección de extractos en un libro titulado Corso di filosofia positiva (Padua: RADAR, 190 p.), con introducción, traducción y comentarios de Ludovico Geymonat y Mario Quaranta. Y en ese mismo año de 1967 ve la luz la más amplia y significativa traducción del Cours, esto es, la que se ocupa de las quince últimas lecciones (las doce relativas a la física social y las tres correspondientes a las conclusiones generales de la obra), publicadas con el título homónimo de Corso di filosofia positiva (Turín: UTET, 2 vols.: vol. 1., Lezioni 46.-54, 697 p.,; vol. 2., Lezioni 55.-60, 755 p.; $2^{\mathrm{a}}$ ed., 1979; existe una versión digitalizada), con edición e introducción a cargo de Franco Ferrarotti y traducciones de Ezio Zagarese, Marina Maioli y Paola Fiorentini Migliucci. De la importancia de esta obra en la difusión del pensamiento comtiano en Italia da cuenta su reciente reedición, en 2009, en un único volumen titulado metonímicamente Comte (Milán: Mondadori, 1439 p.), dentro de la colección "I classici del pensiero". Además, debemos aludir en este apartado a la publicación, en 1974, de una nueva versión parcial de la obra, otra vez con el título completo 
de la obra original, Corso di filosofia positiva, con traducción, introducción y notas a cargo de Almerino Lunardon (Brescia: La Scuola, XLVIII, 221 p.; $2^{\mathrm{a}}$ ed., 1981.; $3^{\mathrm{a}}$ ed., 1987).

En 1964 se publican dos versiones del Discours sur l'esprit positif. El primero de ellos, íntegro, lleva por título Discorso sullo spirito positivo (Turín [etc.]: Istituto editoriale del Mezzogiorno, 109 p.), con traducción, introducción y notas a cargo de Emanuele Riverso, mientras que la segunda, parcial, aparece como Discorso sullo spirito positivo: parti scelte (Nápoles: Il Tripode, 125 p.; $2^{a}$ ed., 141 p.), con traducción, introducción y notas de Luigi Pinto. La tercera versión italiana es la que publica Antimo Negri en el libro Opuscoli di filosofia sociale e discorsi sul positivismo (Florencia: G. C. Sansoni, XLVIII, 787 p., ya citado), varias veces reeditada con posterioridad, ya en volumen separado, con el título Discorso sullo spirito positivo (Roma; Bari: Laterza, 1985, XXXIX, 129 p. ; $2^{\mathrm{a}}$ ed., Roma: Curcio, 1993; $3^{\mathrm{a}}$ ed., Roma; Bari: Laterza, 2001; $4^{\mathrm{a}}$ ed., Roma; Bari: Laterza, 2003).

Contamos con dos versiones diferentes en lengua italiana del Discours sur l'ensemble du positivisme. La primera de ellas es la que publica Antimo Negri en el volumen, varias veces citado, Opuscoli di filosofia sociale e discorsi sul positivismo (Florencia: G. C. Sansoni, XLVIII, 787 p.). La segunda se publica en 1991 con el título Discorso sull'insieme del positivismo (Nápoles: Il Tripode, 1991, 111 p.), con introducción, traducción y notas a cargo de Anna Maria Nieddu.

En 1882 Walter Congreve traduce al italiano Catechismo positivista; ossia, Esposizione sommaria della religione universale in tredici conversazioni sistematiche fra una donna ed un prete dell'umanità (Sanremo: Biancheri, 383 p.), con prólogo de Pierre Laffitte, en la que parece ser, y durante muchos años además, la única versión a esta lengua de cualquiera de las obras de Auguste Comte.

Tenemos que hacer nuevamente referencia a una serie de folletos y breves selecciones textuales publicadas en lengua italiana. Mario Quaranta edita y comenta en 1975 una pequeña selección de extractos de obras comtianas en su obra Previsione e conoscenza: testi filosofici commentati (Treviso: Libreria editrice Canova, 282 p.). En 1990 Fabrizio Battistelli se encarga de la edición de Marte e Mercurio: sociologia dell' organizzazione militare (Milán: Franco Angeli, 563 p.), una obra en la que se incluyen una serie de textos sociológicos clásicos, entre los cuales nos interesa particularmente uno, titulado "Decadenza e trasformazione dell' elemento militare", por extractar el punto de vista de nuestro filósofo respecto al papel del ejército en el devenir social. Por último, Stefania Mariani publica en 1999 Comte. Dizionario delle idee: scienza, politica, morale (Roma: Editori riuniti, XXIII, 111 p.), una breve compilación de extractos tomados del conjunto de la obra comtiana a partir de voces como "Amore", "Anarchia intellettuale", "Animali", "Civiltà", "Classificazione delle scienze", "Comunismo e positivismo", "Divisione del lavoro", "Metodo comparativo", "Morale", "Positivismo", "Scienza", "Teologico", "Vita industriale" o "Vivisezione". 


\section{TRADUCCIONES EN LENGUA RUSA}

El balance de traducciones de la obra comtiana en ruso es relativamente pobre, teniendo en cuenta la trascendencia que esta lengua tiene en la historia intelectual contemporánea. Probablemente la confrontación entre positivismo y marxismo-leninismo ha tenido su correlato en la historia editorial de las traducciones comtianas en dicha lengua: las primeras versiones de textos originales de Auguste Comte, las de Nikolái Losky y las de I. A. Šapiro, son publicadas en fechas relativamente tempranas, entre 1899 y 1913, pero durante el largo periodo soviético no se publica ni una sola nueva versión al ruso de ningún texto comtiano. Sólo a partir de 1996, y particularmente en la actualidad, con la reedición digital de las versiones realizadas a principios del siglo XX, se puede decir que se empieza a normalizar la difusión del pensamiento original de Comte en Rusia. Quizás en el curso de los próximos años se promueva la traducción de textos fundamentales como los primeros opúsculos, los volúmenes sociológicos del Cours (curiosamente, sí que está traducida, íntegramente además, las lecciones relativas a las matemáticas), el Système de politique positive, así como la de otros textos menores.

Una vez más, la compilación de extractos del Cours de philosophie positive elaborada por Jules-Émile Rigolage sirve de primera plataforma de difusión del pensamiento comtiano, también en Rusia. En 1898 se traduce al ruso Социология Конта [Sotsiologiya Konta Sociología comtiana] (San Petersburgo: LF Pantaleev), con traducción a cargo de Nikolái Losky. Entre 1900 y 1901 se publica Курс положительной философии: в 6-ти томах [Kurs polozhitel'noy filosofii: v 6-ti tomakh, Curso de filosofía positiva: en 6 volúmenes] (San Petersburgo: E.K. Gart'ye i K, vol. 1: 302 p.; vol. 2: 163 p.). Se trata de la traducción completa, en dos tomos, pero sólo del primer volumen del Cours, relativo a la filosofía matemática, elaborada a partir de la $5^{a}$ edición, idéntica a la primera, editada, anotada e introducida por S. E. Savitch, S. P. Glazenap, O. D. Khvol'son, D. I. Mendeleev, K. A. Timirjazeva, A. S. Lappo-Danilevsky, I. M. Graves y N. Losky, y un prólogo de N. I. Kareeva.

En 1910 se publica por primera vez en ruso la obra Катехизис промышленников (третья тетрадь) или система позитивной политики [Katekhizis promyshlennikov (tret'ya tetrad') ili sistema pozitivnoy politiki; Catecismo de Industriales (tercer libro) o la positiva política, de Saint-Simon], en una colección que la editorial dedica ex profeso a los pioneros del positivismo (San Petersburgo: s.e., "Iz naslediya mirovoy filosofskoy mysli: sotsial'naya filosofiya", $\left.n^{\circ} 1-2\right)$. En esa misma serie se publican nuevamente, entre 1912 y 1913, las dos primeras lecciones del Cours, otra vez con el título genérico de Курс позитивной философии [Kurs pozitivnoy filosofii; Curso de filosofía positiva] (San Petersburgo: s.e.; "Iz naslediya mirovoy filosofskoy mysli: sotsial'naya filosofiya", $\mathrm{n}^{\circ} 4-5$ ).

También en 1910 se publica la primera versión en ruso del Discours sur l'esprit positif, con el título Дух позитивной философии. (Слово о положительном мышлении) [Dukh pozitivnoy filosofii. (Slovo o 
polozhitel'nom myshlenii)] (San Petersburgo: Izdatel'stvo «Ve囚stnika Znanija» - V. V. Bitnera-, 76 p., IV), traducido por I. A. Šapiro y prologado e introducido

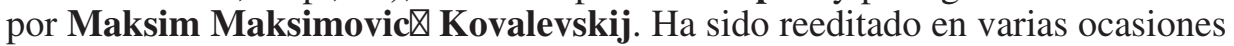
(San Petersburgo: Lab. metafiz. issled. pri filos. fak. SPBGU, XIV, 146 p., con una introducción a cargo de Boris G. Sokolov; Rostov-na-Donu: Feniks, 2003; Moscú: LIBROKOM, 2011, 80 p.).

En 1912 se edita la versión rusa del Discours sur l'ensemble du positivismo, titulada Общий обзор позитивизма [Obshchiy obzor pozitivizma], también traducido por I. A. Shapiro y editado por E. L. Radlova, reeditado ya en dos ocasiones en el curso de los últimos años (Moscú: LIBROKOM, 2010, 2012, 296 p.; existe edición digital de la obra).

En los años 1920 I. V. Markusona traduce la obra del sociólogo alemán Werner Sombart Sotsiologiya [Социология, Sociología] (Leningrado: Mysl'), una antología de textos sociológicos clásicos que incluye, entre otros, extractos de la «la parte dogmática de la filosofía social de [la edición alemana publicada en] Jena en 1907», esto es, del cuarto volumen del Cours, que es traducida como Основные законы социальной динамики, или общая теория естественного прогресса человечества [“Osnovnyye zakony sotsial'noy dinamiki, ili obshchaya teoriya yestestvennogo progressa chelovechestva", "Leyes básicas de la dinámica social, o la teoría general del progreso natural de la humanidad", pp. 15-30], con una nota sobre el autor.

Ya en 1996 se edita una compilación de textos clásicos de la sociología decimonónica europea con el título Западно-европейская соииология XIX века: тексты:: О. Конт, Д.С. Милль, Г. Спенсер [Zapadno-yevropeyskaya sotsiologiya XIX veka: teksty: O. Kont, D.S. Mill', G. Spenser; Textos occidentales decimonónicos de la sociología europea: Comte, Mill, Herbert Spencer] (Moscú: Mezhdunarodnyy universitet biznesa i upravleniya, 352 p.), editada bajo la responsabilidad de Vladimir Ivanovich Dobrenkov. Por último, A. O. Boronoev e I. A. Golosenko publican en 1998 una obra conmemorativa del segundo centenario del nacimiento de Auguste Comte, Огюст Конт. К 200-летию со дня рождения [Ogyust Kont. K 200-letiyu so dnya rozhdeniya] (San Petersburgo: Petropolis, 225 p.), que incluye la traducción de extractos de obras originales del autor.

\section{TRADUCCIONES EN LENGUA JAPONESA}

La producción, ciertamente rica y continuada en el tiempo, de versiones en japonés de textos originales de Auguste Comte es quizás una de las aportaciones más inesperadas y sorprendentes del presente estudio bibliográfico. En efecto, resulta ciertamente curioso comprobar que cualquier lector japonés tiene a su disposición una cantidad nada despreciable de publicaciones en su lengua materna en las que se reproducen extractos significativos de la filosofía comtiana. En esta labor destacan autores como Hori Hidehiko o Juri Tanabe, pero sin duda, por su actualidad y por su empeño sobresale particularmente el rol que Takashi Sugimoto está jugando en la divulgación del pensamiento 
comtiano en lengua japonesa.

En 1928 Sanshiro $\bigotimes$ Ishikawa publica la traducción al japonés de una selección de textos sobre filosofía positiva con el título 世界大思想全集. 第 25,26 卷, 実証哲学. 上, 下卷 [Sekai dai shisō zenshū. Dai 25, 26 卷, Jisshōtetsugaku.-Jō,-ka 卷] (Tokio: Shunju囚sha, reed. en 1931 y 1934). Otra selección de textos comtianos es la que se edita en 1960 en la obra コントス ペンサー [Konto, Supensā] (Tokio: Kawade Shobō Shinsha, 344 p.), en la que Hori Hidehiko incluye además extractos sociológicos de la obra de Herbert Spencer. La antología ha sido reimpresa en varias ocasiones (Tokio: Chūō Kōronsha, 1970, 1980 y 1999, 494 p.). De semejante factura es el volumen 世 界思想教養全集. 10 [Sekai shisō kyōyō zenshū. 10.] (Tokio: Kawade Shobo Shinsha, 397 p.), de 1964, en el que Takashi Ide incluye una amplia selección de textos filosóficos de Auguste Comte y Ludwig Feuerbach. Mucho más reciente, y completa, es la antología que Takashi Sugimoto traduce, comenta y publica en 2013 en la obra 科学=宗教という地平 [Kagaku ikoru shukyo to iu chihei] (Tokio: Hakusuisha, 2013, 217 p.), en la que se incluyen textos sobre ciencia y religión. Precisamente este mismo autor es el autor de la traducción del "Appendice général du Système de Politique Positive, contenant tous les opuscules primitifs de l'auter sur la philosophie sociale", publicado también en 2013 en un volumen que lleva por título ソシオロジーの起源へ [Soshioroji no kigen e.] (Tokio: Hakusuisha, 249 p.).

Del "opúsculo fundamental" tenemos al menos otras dos versiones: la primera de ellas es de 1938, se titula 社会の再組織について [Shakai no saisoshiki ni tsuite] (Tokio: Iwanami Shoten, 1938, 147 p.) y la ejecuta Ken'ichi Tobisawa; la segunda es la que publica Bungo Tsuchiya en 1949 en un volumen titulado 社会の再組織について [Shakai no saisoshiki ni tsuite] (Tokio: So囚gensha, 189 p.).

La primera traducción al japonés del Discours sur l'esprit positif también data de 1938 y se titula 実証的精神論 [Jisshoteki seishinron] (Tokio: Iwanami Shoten, 228 p.; reed., Tokio: Shinano Shobo, 293 p.), figurando Juri Tanabe como traductor del texto. Existe una segunda versión de esta misma obra, publicada en este caso en 1948 con el título 実証精神叙說 [Jisshō seishin josetsu] (Tokio: So囚gensha, 148 p.), con traducción a cargo de Takaji Hirayama.

\section{TRADUCCIONES EN OTRAS LENGUAS}

Si hubiera que destacar alguna peculiaridad de las traducciones holandesas de la obra comtiana esta sería, sin duda, su carácter pionero. En efecto, la primera traducción conocida de cualquier obra de Auguste Comte a cualquiera de las lenguas del mundo es la que Josué L'Ange Huet edita en 1846 con el título Algemeene grondslagen der stellige wijsbegeerte: uitgave door eenige voorstanders der stellige wijsbegeerte ('s-Gravenhage: Belinfante, X, 111 p.; existe edición electrónica). Apenas un año después, en 1847, se publica De Nieuwe wijsgeerige school (Utrecht: s.n., 48 p.), una breve selección de 
textos tomados del Cours. La tercera referencia, mucho más reciente, es la correspondiente a la traducción del Discours sur l'esprit positif, publicada en 1979 bajo el título Het positieve denken (Ámsterdam: Boom Meppel, 172 p.), con traducción de Henriët Plantenga, e introducción y notas a cargo de J. M. M. de Valk.

En catalán contamos con la traducción del Discours sur l'esprit positif, así como la de las dos primeras lecciones del Cours, publicadas en 1982 en un volumen titulado Discurs sobre lésperit positiu precedit de les dues primeres lliçons del Curs de filosofía positiva (Barcelona: Laia, 250 p.), con edición a cargo de Joan Rovira y traducción de Jaume Melendres.

En 1875 se realiza la primera traducción al sueco del Discours sur l'esprit positif, publicada con el título Framställning öfver den positive anden (Estocolmo: Ebeling \& K., VI, 116 p.), con introducción a cargo de Anton Nyström. Este mismo autor, también en 1875, se encarga de la edición de la primera versión en lengua sueca del Calendrier positiviste, en este caso con el título Positivistisk kalender eller tablå öfver mensklighetens utveckling (Estocolmo, s.e.: 1 tabla, 2 p.). Apenas seis años después, en 1881, ve la luz una segunda versión, titulada en este caso A. Comte positivistiska kalender eller konkreta tablå öfver mensklighetens förberedande utveckling, jemet tillägg af de särdkildt för Sverige betydelsefulla personligheterna (Estocolmo: s.e.). En ese mismo año de 1881 ve la luz la publicación de Religionens väsende och mensklighetsbegreppet (Estocolmo: s.e.), acompañado de una introducción firmada por L. N. [a la sazón, Louise Hamilton]. En 1896 aparece a su vez Allmän öfversigt af positivismen (Estocolmo: Bonnier, 335 p.), traducción al sueco del Discours sur l'ensemble du positivisme que incluye un prólogo a cargo de Anton Nyström y una introducción firmada por L. N. y C. B. Tras un larguísimo paréntesis en el que parece desaparecer el interés editorial sueco por la publicación de traducciones de la obra comtiana, Otto Mannheimer publica en 1979 Om positivismen [Sobre el positivismo] (Göteborg: Bokförlaget Korpen, 92 p.), la primera versión sueca del Discours preliminaire sur l'esprit positif. Esta misma traducción aparece nuevamente reimpresa en un volumen en el que se recompilan textos clásicos de la sociología que lleva precisamente por título Tre klassiska texter (Göteborg: Bokförlaget Korpen, pp. 5-120), y en el cual, además de la citada obra extractada de Auguste Comte, se incluyen otras tantas traducciones de sendos escritos de Émile Durkheim y Max Weber.

En 1991 se publica en danés Religionsvidenskabelig grunduddannelse: forskningshistorie og metodologi (Copenhague, 137 p.), una monografía que incluye la traducción de algunos extractos originales relativos al pensamiento de Auguste Comte, entre otros autores, sobre religión.

En 1961 ve la luz una nueva traducción, en esta ocasión al polaco, del varias veces citado resumen del Cours elaborado por Jules-Émile Rigolage: Metoda pozytywna $v$ szesnastu wykladach [El método positivo en dieciséis lecciones] (Varsovia: Panstwowe Wydawnictwo Naukowe), con un prólogo a cargo de Wanda Wojciechowska. Por otro lado, tenemos que hacer asimismo referencia a la publicación, en 1936, de la primera edición en polaco del Discours sur 
l'esprit positif, titulada Rozprawa o duchu filozofii pozytywnej (Varsovia: Wydaw. Kasy im. Mianowskiego-Instututu Popierania Nauki, XVI, 129 p.), con traducción a cargo de J. K. Esta versión se reedita en 2001 (Kęty: Antyk, 93 p.), 2003 (Varsovia: Altaya Polska, 160 p.) y 2009 (Varsovia: Hachette, 167 p.). Asimismo, en 1973 se publica una nueva traducción del Discours sur l'esprit positif, acompañada además de la primera versión polaca del Discours sur l'ensemble du positivisme, en un volumen titulado Rozprawa o duchu filozofii pozytywnej; Rozprawa o catoksztatcie pozytywizmu (Varsovia: Panstwowe Wydawnictwo Naukowe, XLII, 622 p.), editado, introducido y anotado por Barbara Skarga y traducido por Wanda Wojciechowska y la propia Barbara Skarga.

En cuanto a las traducciones a la lengua checa, hay que señalar que en 1889 se publica Sociologie (Praga: Bursík \& Kohout, 458, XII p.; 2a ed., 1893), la enésima versión del libro extractado por Jules-Émile Rigolage, con traducción a cargo de Rudolf Brejcha. Esta misma obra se reedita en 1927 (Praga: Orbis, 466 p.), acompañada de una introducción de Emanuel Chalupný. En 1946 se publica el folleto Rozumová anarchie a sociální zla [Anarquía intelectual y males sociales] (Brno: Nákladem Masarykovy sociologické spolecnosti v Brne, 23 p.), a partir de extractos del tercer volumen de la edición original del Cours (1839, pp. 91-138), traducidos "libremente del francés", tal y como se indica en los títulos de crédito de la obra, por Juliana Obrdlíková. Además, habría que mencionar la edición, en 1969, Auguste Comte: výbor z díla [A. Comte: antología] (Praga: Vysoká škola politická ÚV KSC, 172 p.), de la que se encarga Miroslav Hutar.

En búlgaro contamos con dos publicaciones de traducciones de textos originales de Comte, surgidas ambas probablemente al hilo del bicentenario de su nacimiento. La primera de ellas se la debemos al trabajo de Evgenija Grekova, data de 1998, se titula Socialnata nauka (Sofía: Glorija Mundi, 264 p.) y en realidad constituye una versión de La science sociale (París: Éditions Gallimard, 1972, 308 p.), uno de los más conocidos volúmenes antológicos publicados en lengua francesa con extractos de diversas obras comtianas; la segunda, que ve la luz en 2000, es la traducción del Discours sur l'esprit positif realizada por Todorka Mineva y titulada Traktat vărhu pozitivnija duh (Sofía: Sonm, 136 p.).

Debemos mencionar asimismo la publicación, en 1979, de la versión húngara del Plan des travaux scientifiques nécessaires pour réorganiser la société y del Discours sur l'esprit positif, que Gábor Berényi realiza y edita en un único volumen con el título A pozitiv szellem: két értekezés (Budapest: M. Helikon, 427 p.).

En lengua rumana hemos localizado una única traducción, la de del Calendrier, publicada en 1931 sin mención al traductor responsable y titulada Calendarul Positivist: Potrivit pe romineste en o introducere despre calendare in general si despre cel pozitivist san stientific in special do Dr. P. Zozin (s.l.: IASI - Institutal de Arte Grafice N. V. Stefane e G ${ }^{\circ}, 32$ p.).

En cuanto al croata, también disponemos de una única versión de una obra

EMPIRIA. Revista de Metodología de Ciencias Sociales. N. ${ }^{\circ}$ 31, mayo-agosto, 2015, pp. 173-198. ISSN: 1139-5737, DOI/empiria.29.2014.12942 
de Auguste Comte, pero muy significativa, concretamente la que se publica en 1989 con el título Kurs pozitivne filozofije (Nikšic: Univerzitetska Rijec, 307 p.; existe edición digital), en la cual se reproduce íntegramente el cuarto volumen de la edición original del Cours (lecciones $46^{\mathrm{a}}$ a $51^{\mathrm{a}}$ ), con prólogo y traducción de Radomir D. Lukic y edición a cargo de Ratko Božović y Dragan Vukčević. Además, también cabe referirse aquí a la publicación en 1962, en lengua serbia, de una traducción de las dos primeras lecciones del Cours de philosophie positive con el título equivalente de Kurs pozitivne filozofije: dva uvodna predavanja (Belgrado: Kultura, 62 p.), con prólogo de Radmila Šajković y traducción de Vlado Drašković. En lengua macedonia es posible leer una versión relativamente extensa del Cours, editada en 2007 con un prólogo de Kole Jovanovski y traducción a cargo de Filip Iliev, y titulada Kurs po pozitivna filozofija (Skopje: Az-Buki, 429 p.).

En lengua griega hemos localizado dos únicas referencias bibliográficas: la primera de ellas remite a un volumen en el que se extracta el Cours,

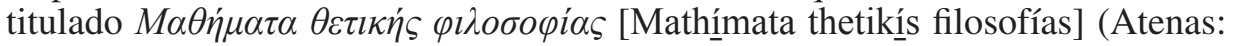
Anagnostidis, s.f., 207 p.), con traducción a cargo de Janis lo Skokko y edición de K. Metrinou; la segunda es la relativa a la publicación en 2000 de

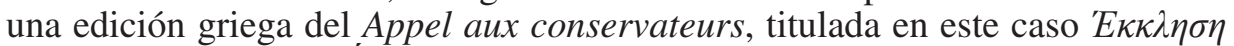

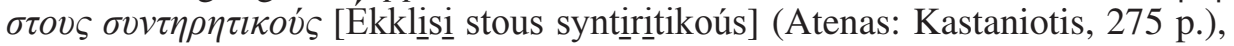
traducido por Kostis Papagiorgis.

En 2001 se publica una versión muy parcial en lengua turca del Cours de philosophie positive, con el título Pozitif felsefe kurslarl (Estambul: Sosyal Yayınlar, 161 p.), con traducción de Erkan Ataçay. En 1952 ve la luz Pozitivizm ilmihali (Estambul: Milli Eğitim, LXXIII, 396 p.; reed., 1986) la primera traducción a la lengua turca del Catéchisme positiviste, realizada por Peyami Erman. Y en 2003 aparece publicado Pozitif felsefeye giris (Ankara: Yeryuzu Yayinevi, 79 p.), traducido por Suleyman Sari.

La producción editorial en lengua china en la que se refiere a la publicación de traducciones de la obra comtiana palidece, sin duda, si la comparamos con la relativa abundancia de referencias que hemos localizado para el caso del japonés. Son apenas dos: las versiones chinas de los dos Discours. En efecto, en 1996 se publica el Discours sur l'esprit positif con el título 论实证精神 [Lun shi zheng jing shen] (Beijing: Shang wu yin shu guan, 95 p.; reeds.: 1999, 2009; existe edición digital) y traducción a cargo de Jianhua Huang, y existe también una version china del Discours sur l'ensemble du positivisme, más antigua, ejecutada por Gan Xiao en 1938 y que ve la luz con el título 實證主義概觀 [Shi zheng zhu yi gai guan, Visión general del positivismo] (Changsha: Shang wu yin shu guan, 433 p.; varias reeds.: Tai bei shi: Shang wu, 1965, 1966, 1973, 425 p.; Beijing: Quanguo tushuguan wenxian suowei zhongxin, 2005).

Para finalizar, tenemos que mencionar la traducción al coreano, en 2001, del Discours preliminaire sur l'ensemble du positivisme, con el título 실증주의 서설 [Silchŭngjuŭi sŏsŏl] (Kyŏnggi-do P'aju-si: Han'gilsa, 467 p.), con traducción a cargo de Kim Chŏm-sŏk. 


\section{BALANCE FINAL}

La historia de las traducciones de la obra de Auguste Comte a las diferentes lenguas del mundo pone de manifiesto la importancia crucial del pensamiento del fundador del positivismo en la historia intelectual contemporánea. Desde luego, se puede asegurar, sin temor a error, que se trata de uno de los filósofos cuya obra ha sido más traducida, y a un mayor número de lenguas diferentes además, a lo largo de los últimos ciento cincuenta años. Desde la pionera versión de Josué L'Ange Huet en holandés, que data de 1846, hasta las traducciones publicadas en 2013 por Takashi Sugimoto en japonés, hemos asistido a un rico proceso editorial que ha permitido la difusión del pensamiento comtiano a través de cinco continentes y una gran cantidad de culturas intelectuales. En cada una de ellas, e incluso en cada uno de los países en los que Comte ha tenido cierta acogida, se ha producido una historia editorial e intelectual compleja, con sus luces y también con sus sombras, pero de lo que no cabe duda es que la actividad translatoria realizada por varias decenas de traductores en el curso de todos estos años ha permitido un mejor y más profundo conocimiento de las aportaciones de Auguste Comte a la historia de la filosofía. Y más teniendo en cuenta que muchos de esos textos traducidos, algunos realmente antiguos, están a disposición de cualquier usuario interesado incluso en versión digital, con todas sus consecuencias, incluida la facilidad de acceso documental y/o lectura desde un e-book o tablet.

De lo que no cabe duda, finalmente, es que la relación bibliográfica de las traducciones de la obra de Auguste Comte a las principales lenguas del mundo que acabamos de presentar puede servir para hacerse una idea cabal de su riqueza y gran variedad, si bien, como en cualquier otro ámbito, el futuro seguirá estando preñado de posibilidades de mejora: todavía quedan muchas lagunas por rellenar, muchas viejas traducciones que revisar, muchas nuevas versiones que acometer. 